\title{
Berends-Giele recursions and the $\mathrm{BCJ}$ duality in superspace and components
}

\author{
Carlos R. Mafra ${ }^{a, b}$ and Oliver Schlotterer ${ }^{c}$ \\ ${ }^{a}$ Institute for Advanced Study, School of Natural Sciences, \\ Einstein Drive, Princeton, NJ 08540, U.S.A. \\ ${ }^{b}$ DAMTP, University of Cambridge, \\ Wilberforce Road, Cambridge, CB3 0WA, U.K. \\ ${ }^{c}$ Max-Planck-Institut für Gravitationsphysik, Albert-Einstein-Institut, \\ Am Muehlenberg, 14476 Potsdam, Germany \\ E-mail: mafra@ias.edu, olivers@aei.mpg.de
}

\begin{abstract}
The recursive method of Berends and Giele to compute tree-level gluon amplitudes is revisited using the framework of ten-dimensional super Yang-Mills. First, we prove that the pure spinor formula to compute SYM tree amplitudes derived in 2010 reduces to the standard Berends-Giele formula from the 80s when restricted to gluon amplitudes and additionally determine the fermionic completion. Second, using BRST cohomology manipulations in superspace, alternative representations of the component amplitudes are explored and the Bern-Carrasco-Johansson relations among partial tree amplitudes are derived in a novel way. Finally, it is shown how the supersymmetric components of manifestly local BCJ-satisfying tree-level numerators can be computed in a recursive fashion.
\end{abstract}

KEYwORDs: Superspaces, Superstrings and Heterotic Strings

ARXIV EPRINT: 1510.08846 


\section{Contents}

1 Introduction 1

1.1 Summary of results on the Berends-Giele recursion 2

1.2 Summary of results on the BCJ duality 3

2 Review 4

2.1 Berends-Giele recursion relations 4

2.2 Super Yang-Mills superfields in ten dimensions 5

2.2.1 Simplifying component expansions with superfield gauge transformations 6

2.3 The pure spinor superspace formula for SYM tree amplitudes $\quad 7$

3 The supersymmetric completion of the Berends-Giele formula 7

$\begin{array}{lll}3.1 & \text { Bosonic Berends-Giele current from superfields } & 7\end{array}$

3.2 Supersymmetric Berends-Giele amplitude from the pure spinor formula 8

$\begin{array}{lll}3.3 & \text { Divergent propagators and their cancellation } & 9\end{array}$

$\begin{array}{lll}3.3 .1 & \text { In components } & 9\end{array}$

$\begin{array}{lll}\text { 3.3.2 In pure spinor superspace } & 9\end{array}$

3.4 Short representations and BRST integration by parts 9

$\begin{array}{ll}3.5 & \text { The generating series of tree-level amplitudes } \\ \end{array}$

4 BCJ relations from the cohomology of pure spinor superspace 11

4.1 Kleiss-Kuijf relations from symmetries of Berends-Giele currents 11

$\begin{array}{lll}4.2 & \text { BCJ relations from the BRST cohomology } & 12\end{array}$

$\begin{array}{lll}\text { 4.2.1 Berends-Giele currents in BCJ gauge } & 12\end{array}$

$\begin{array}{lll}4.2 .2 & \text { Four- and five-point BCJ relations } & 13\end{array}$

$\begin{array}{lll}\text { 4.2.3 Higher-point BCJ relations } & 14\end{array}$

$\begin{array}{lll}4.3 & \text { Component form of BCJ numerators } & 15\end{array}$

$\begin{array}{lll}\text { 4.3.1 Local multiparticle polarizations } & 15\end{array}$

$\begin{array}{lll}\text { 4.3.2 Higher multiplicity } & 17\end{array}$

5 Conclusion and outlook $\quad 17$

\section{Introduction}

Ten-dimensional super Yang-Mills (SYM) provides a simplified description of maximally supersymmetric gauge theories [1]. On the one hand, its spectrum comprises just a gluon and a gluino which automatically cover the scalars in lower-dimensional formulations [2]. On the other hand, pure spinors allow to formulate the on-shell conditions as a cohomology 
problem [3, 4], and the BRST operator in the associated pure spinor superspace powerfully embodies gauge invariance and supersymmetry [5]. This framework naturally appears in the manifestly super Poincaré-covariant quantization of the superstring [5].

Using a confluence of string-theory techniques and field-theory intuition, scattering amplitudes in ten-dimensional SYM have been compactly represented in pure spinor superspace [6-8]. This construction crucially rests on the notion of multiparticle superfields [9] which were motivated by superstring computations [10-14]. Multiparticle superfields collect the contributions of tree-level subdiagrams at arbitrary multiplicity and can be flexibly attached to multiloop diagrams, see [8] for a two-loop application.

In a companion paper [15], the construction of multiparticle superfields and their expansion in the Grassmann variable $\theta^{\alpha}$ of pure spinor superspace have been tremendously simplified. In the following, we will revisit tree-level amplitudes in the light of the new theta-expansions and in particular:

- recover and supersymmetrize the Berends-Giele recursion for gluonic tree amplitudes

- present a simplified component realization of the BCJ color-kinematics duality, along with a new superspace proof for the closely related BCJ relations.

\subsection{Summary of results on the Berends-Giele recursion}

The theta-expansions of ten-dimensional multiparticle superfields have recently [15] been simplified using supersymmetric Berends-Giele currents which generalize the gluonic currents defined by Berends and Giele [16]. Using these simplified expansions, the pure spinor superspace formula to compute ten-dimensional color-ordered SYM amplitudes at tree level [6],

$$
A^{\mathrm{SYM}}(1,2, \ldots, p, p+1)=\left\langle E_{12 \ldots p} M_{p+1}\right\rangle,
$$

will be explicitly evaluated in components and shown to be

$$
A^{\mathrm{SYM}}(1,2, \ldots, p, p+1)=s_{12 \ldots p}\left(\mathfrak{e}_{12 \ldots p} \cdot \mathfrak{e}_{p+1}\right)+k_{12 \ldots p}^{m}\left(\mathcal{X}_{12 \ldots p} \gamma_{m} \mathcal{X}_{p+1}\right) .
$$

The superfields $E_{12 \ldots p}$ and $M_{p+1}$ will be introduced in section 2.3 , and the square of the momentum $k_{12 \ldots p}^{m} \equiv k_{1}^{m}+k_{2}^{m}+\cdots+k_{p}^{m}$ is denoted by $s_{12 \ldots p}$. Moreover, $\mathfrak{e}_{12 \ldots p}^{m}$ and $\mathcal{X}_{12 \ldots p}^{\alpha}$ in (1.2) denote the component Berends-Giele currents which depend on the gluon and gluino polarizations $e_{i}^{m}, \chi_{i}^{\alpha}$ as well as light-like momenta $k_{i}^{m}$ associated with legs $i=1,2, \ldots, p$. Finally, $m=0, \ldots, 9$ and $\alpha=1, \ldots, 16$ are vector and Weyl-spinor indices of the Lorentz group $\mathrm{SO}(1,9)$.

After setting the fermionic fields to zero, the first term in (1.2) will be shown to reproduce the gluonic Berends-Giele formula [16],

$$
A^{\mathrm{YM}}(1,2, \ldots, p+1)=s_{12 \ldots p}\left(J_{12 \ldots p} \cdot J_{p+1}\right)
$$

making (1.2) its supersymmetric generalization for ten-dimensional SYM. 
Furthermore, the same Berends-Giele currents $\mathfrak{e}_{12 \ldots p}^{m}$ and $\mathcal{X}_{12 \ldots p}^{\alpha}$ together with a fieldstrength companion $\mathfrak{f}_{12 \ldots p}^{m n}$ will be shown to yield economic and manifestly cyclic representations of SYM amplitudes such as

$$
\begin{aligned}
A^{\mathrm{SYM}}(1,2,3,4,5)= & \frac{1}{2}\left(\mathfrak{e}_{12}^{m} \mathfrak{f}_{34}^{m n} \mathfrak{e}_{5}^{n}+\mathfrak{e}_{34}^{m} \mathfrak{f}_{5}^{m n} \mathfrak{e}_{12}^{n}+\mathfrak{e}_{5}^{m} \mathfrak{f}_{12}^{m n} \mathfrak{e}_{34}^{n}\right) \\
& +\left(\mathcal{X}_{12} \gamma_{m} \mathcal{X}_{5}\right) \mathfrak{e}_{34}^{m}+\left(\mathcal{X}_{34} \gamma_{m} \mathcal{X}_{12}\right) \mathfrak{e}_{5}^{m}+\left(\mathcal{X}_{5} \gamma_{m} \mathcal{X}_{34}\right) \mathfrak{e}_{12}^{m}+\operatorname{cyclic}(12345)
\end{aligned}
$$

streamlining the earlier approach in [17] based on the above $J_{12 \ldots}^{m}$.

Using the generating series of supersymmetric Berends-Giele currents discussed in $[15,18]$, it will be shown that the generating series of ten-dimensional SYM tree-level amplitudes takes a very simple form,

$$
\left.\operatorname{Tr}\left(\frac{1}{4} \mathbb{F}_{m n} \mathbb{F}^{m n}+\left(\mathbb{W} \gamma^{m} \nabla_{m} \mathbb{W}\right)\right)\right|_{\theta=0}=\sum_{n=3}^{\infty} \frac{n-2}{n_{i_{1}, i_{2}, \ldots, i_{n}}} \sum \operatorname{Tr}\left(t^{i_{1}} t^{i_{2}} \ldots t^{i_{n}}\right) A^{\mathrm{SYM}}\left(i_{1}, i_{2}, \ldots, i_{n}\right)
$$

Note that the left-hand side of (1.5) matches the ten-dimensional SYM Lagrangian evaluated on the generating series $\mathbb{F}^{m n}(x, \theta=0)$ and $\mathbb{W}^{\alpha}(x, \theta=0)$ defined below.

\subsection{Summary of results on the BCJ duality}

The virtue of the simplified theta-expansions in [15] can be reconciled with a manifestation of the duality between color and kinematics due to Bern, Carrasco and Johansson (BCJ) [19] (see [20] for a review). A concrete tree-level realization of the BCJ duality was given in [21] at any multiplicity, based on local numerators in pure spinor superspace. The components are accessible through the zero-mode treatment in [22], but we will present a significantly accelerated approach where the zero-mode manipulations are trivialized.

The BCJ duality immediately led to the powerful prediction that only $(n-3)$ ! permutations of SYM tree-level subamplitudes (1.2) are linearly independent [19]. This basis dimension was later derived from the monodromy properties of the string worldsheet [23, 24], by the field-theory limit of the $n$-point superstring disk amplitude $[13,25]$ and by BCFW on-shell recursions in field theory [26]. In addition to these proofs, the following explicit BCJ relations among color-ordered amplitudes will be obtained from pure spinor cohomology arguments,

$$
\sum_{i=1}^{|A|} \sum_{j=1}^{|B|}(-1)^{i-j} s_{a_{i} b_{j}} A^{\mathrm{SYM}}\left(\left(a_{1} \ldots a_{i-1} ш a_{|A|} \ldots a_{i+1}\right), a_{i}, b_{j},\left(b_{j-1} \ldots b_{1} ш b_{j+1} \ldots b_{|B|}\right), n\right)=0,
$$

where the words $A=a_{1} a_{2} \ldots a_{|A|}$ and $B=b_{1} b_{2} \ldots b_{|B|}$ have total length $|A|+|B|=n-1$. The shuffle product $\omega$ is defined recursively as

$$
\emptyset ш A=A ш \emptyset=A, \quad A ш B \equiv a_{1}\left(a_{2} \ldots a_{|A|} \uplus B\right)+b_{1}\left(b_{2} \ldots b_{|B|} ш A\right),
$$

where $\emptyset$ denotes the case when no "letter" is present. 


\section{Review}

\subsection{Berends-Giele recursion relations}

In the 80s, Berends and Giele proposed a recursive method to compute color-ordered gluon amplitudes at tree level using multiparticle currents $J_{P}^{m}$ defined $^{1}$ as [16]

$$
J_{i}^{m} \equiv e_{i}^{m}, \quad s_{P} J_{P}^{m} \equiv \sum_{X Y=P}\left[J_{X}, J_{Y}\right]^{m}+\sum_{X Y Z=P}\left\{J_{X}, J_{Y}, J_{Z}\right\}^{m},
$$

where $e_{i}^{m}$ denotes the polarization vector of a single-particle gluon, $P=12 \ldots p$ encompasses several external particles, and the Mandelstam invariants are

$$
s_{P} \equiv \frac{1}{2} k_{P}^{2}, \quad k_{P}^{m} \equiv k_{1}^{m}+k_{2}^{m}+\cdots+k_{p}^{m} .
$$

The notation $\sum_{X Y=P}$ in (2.1) instructs to deconcatenate $P=12 \ldots p$ into non-empty words $X=12 \ldots j$ and $Y=j+1 \ldots p$ with $j=1,2, \ldots, p-1$ and the obvious generalization to $\sum_{X Y Z=P}$. The brackets $[\cdot, \cdot]^{m}$ and $\{\cdot, \cdot, \cdot\}^{m}$ are given by stripping off one gluon field (with vector index $m$ ) from the cubic and quartic vertices of the Yang-Mills Lagrangian,

$$
\begin{aligned}
{\left[J_{X}, J_{Y}\right]^{m} } & \equiv\left(k_{Y} \cdot J_{X}\right) J_{Y}^{m}+\frac{1}{2} k_{X}^{m}\left(J_{X} \cdot J_{Y}\right)-(X \leftrightarrow Y) \\
\left\{J_{X}, J_{Y}, J_{Z}\right\}^{m} & \equiv\left(J_{X} \cdot J_{Z}\right) J_{Y}^{m}-\frac{1}{2}\left(J_{X} \cdot J_{Y}\right) J_{Z}^{m}-\frac{1}{2}\left(J_{Y} \cdot J_{Z}\right) J_{X}^{m} .
\end{aligned}
$$

The Berends-Giele currents $J_{P}^{m}$ are conserved [16] and satisfy certain symmetries [27],

$$
k_{P}^{m} J_{P}^{m}=0, \quad J_{A \amalg B}^{m}=0, \quad \forall A, B \neq \emptyset .
$$

The purely gluonic amplitudes are then computed as [16]

$$
A^{\mathrm{YM}}(1,2, \ldots, p, p+1)=s_{12 \ldots p} J_{12 \ldots p}^{m} J_{p+1}^{m} .
$$

For example, the Berends-Giele current of multiplicity two following from (2.1) is

$$
s_{12} J_{12}^{m}=e_{2}^{m}\left(e_{1} \cdot k_{2}\right)-e_{1}^{m}\left(e_{2} \cdot k_{1}\right)+\frac{1}{2}\left(k_{1}^{m}-k_{2}^{m}\right)\left(e_{1} \cdot e_{2}\right)
$$

and leads to the well-known three-point amplitude

$$
A^{\mathrm{YM}}(1,2,3)=s_{12} J_{12}^{m} J_{3}^{m}=\left(e_{1} \cdot e_{2}\right)\left(k_{1} \cdot e_{3}\right)+\operatorname{cyclic}(123) .
$$

Note that the Berends-Giele formula (2.6) as presented in [16] is not supersymmetric, it computes purely gluonic amplitudes.

\footnotetext{
${ }^{1}$ The original definition of $J_{P}^{m}$ in [16] contains the factor $1 / k_{P}^{2}$ instead of $1 / s_{P}$ as adopted here. An overall factor of $\frac{1}{2}$ in (2.3) and (2.4) compensates this difference.
} 


\subsection{Super Yang-Mills superfields in ten dimensions}

SYM in ten dimensions admits a super-Poincare-invariant description in terms of four types of superfields: the spinor potential $\mathbb{A}_{\alpha}(x, \theta)$, the vector potential $\mathbb{A}^{m}(x, \theta)$ and their associated field-strengths $\mathbb{W}^{\alpha}(x, \theta), \mathbb{F}^{m n}(x, \theta)$. They satisfy the following non-linear field equations $^{2}[1]$,

$$
\begin{aligned}
\left\{D_{(\alpha}, \mathbb{A}_{\beta)}\right\} & =\gamma_{\alpha \beta}^{m} \mathbb{A}_{m}+\left\{\mathbb{A}_{\alpha}, \mathbb{A}_{\beta}\right\} \\
{\left[D_{\alpha}, \mathbb{A}_{m}\right] } & =\left[\partial_{m}, \mathbb{A}_{\alpha}\right]+\left(\gamma_{m} \mathbb{W}\right)_{\alpha}+\left[\mathbb{A}_{\alpha}, \mathbb{A}_{m}\right] \\
\left\{D_{\alpha}, \mathbb{W}^{\beta}\right\} & =\frac{1}{4}\left(\gamma^{m n}\right)_{\alpha}{ }^{\beta} \mathbb{F}_{m n}+\left\{\mathbb{A}_{\alpha}, \mathbb{W}^{\beta}\right\} \\
{\left[D_{\alpha}, \mathbb{F}^{m n}\right] } & =\left[\partial^{[m},\left(\mathbb{W} \gamma^{n]}\right)_{\alpha}\right]-\left[\mathbb{A}^{[m},\left(\mathbb{W} \gamma^{n]}\right)_{\alpha}\right]+\left[\mathbb{A}_{\alpha}, \mathbb{F}^{m n}\right] .
\end{aligned}
$$

For later convenience, we use the notation where $\mathbb{K}$ refers to any element of the set containing these superfields,

$$
\mathbb{K} \in\left\{\mathbb{A}_{\alpha}, \mathbb{A}_{m}, \mathbb{W}^{\alpha}, \mathbb{F}^{m n}\right\} .
$$

In the context of scattering amplitudes or vertex operators of the superstring [5], one discards the quadratic terms from (2.9) to obtain the linearized superfields of ten-dimensional $\mathrm{SYM} K_{i} \in\left\{A_{\alpha}^{i}, A_{m}^{i}, W_{i}^{\alpha}, F_{i}^{m n}\right\}$ satisfying

$$
\begin{aligned}
\left\{D_{(\alpha}, A_{\beta)}^{i}\right\} & =\gamma_{\alpha \beta}^{m} A_{m}^{i}, & & \left\{D_{\alpha}, W_{i}^{\beta}\right\}=\frac{1}{4}\left(\gamma^{m n}\right)_{\alpha}{ }^{\beta} F_{m n}^{i} \\
{\left[D_{\alpha}, A_{m}^{i}\right] } & =\left(\gamma_{m} W_{i}\right)_{\alpha}+\left[\partial_{m}, A_{\alpha}^{i}\right], & & {\left[D_{\alpha}, F_{m n}^{i}\right]=\left[\partial_{[m},\left(\gamma_{n]} W_{i}\right)_{\alpha}\right] . }
\end{aligned}
$$

They describe a single gluon and/or gluino which furnishes the $i^{\text {th }}$ leg in the amplitude.

In pursuing compact expressions for superstring scattering amplitudes one is led to a natural multiparticle generalization of the above description, where the singleparticle labels are replaced by "words" $P=123 \ldots p$. In particular, amplitudes can be compactly written in terms of non-local ${ }^{3}$ superfields called Berends-Giele currents $\mathcal{K}_{P} \in\left\{\mathcal{A}_{\alpha}^{P}, \mathcal{A}_{m}^{P}, \mathcal{W}_{P}^{\alpha}, \mathcal{F}_{P}^{m n}\right\}$ encompassing several legs $1,2, \ldots, p$ in an amplitude. They are recursively constructed from linearized superfields in (2.11), and the original expressions in [9] are related to simplified representations in [15] via non-linear gauge transformations. This gauge freedom affects the generating series $\mathbb{K} \in\left\{\mathbb{A}_{\alpha}, \mathbb{A}_{m}, \mathbb{W}^{\alpha}, \mathbb{F}^{m n}\right\}$ of Berends-Giele currents

$$
\mathbb{K}=\sum_{i} \mathcal{K}_{i} t^{i}+\sum_{i, j} \mathcal{K}_{i j} t^{i} t^{j}+\sum_{i, j, k} \mathcal{K}_{i j k} t^{i} t^{j} t^{k}+\cdots
$$

where $t^{i}$ are generators of a non-abelian gauge group. The generating series in (2.12) were shown in [18] to solve the non-linear field equations ${ }^{4}(2.9)$ by the properties of the constituent Berends-Giele currents $\mathcal{K}_{P} \in\left\{\mathcal{A}_{\alpha}^{P}, \mathcal{A}_{m}^{P}, \mathcal{W}_{P}^{\alpha}, \mathcal{F}_{P}^{m n}\right\}$.

\footnotetext{
${ }^{2}$ Our convention for (anti)symmetrizing indices does not include $\frac{1}{2}$, e.g. $\partial^{[m} \gamma^{n]}=\partial^{m} \gamma^{n}-\partial^{n} \gamma^{m}$.

${ }^{3} \mathrm{~A}$ discussion of local multiparticle superfields $K_{P}$ can be found in $[9,15]$.

${ }^{4}$ It should be pointed out that the notion of a generating series which solves the field equations and gives rise to tree amplitudes corresponds to the "perturbiner" formalism [28-30]. This approach has been applied to the self-dual sector of Yang-Mills theory and led to a generating series of MHV amplitudes, see [31] for a supersymmetric extension. However, the generic Yang-Mills amplitudes have never been obtained this way (see also [32]). We thank Nima Arkani-Hamed for pointing out these references.
} 


\subsubsection{Simplifying component expansions with superfield gauge transforma- tions}

The aforementioned gauge freedom of the generating series (2.12) allows to tune the thetaexpansion of the multiparticle supersymmetric Berends-Giele currents such that [15]

$$
\mathcal{A}_{\alpha}^{P}(x, \theta)=\left(\frac{1}{2}\left(\theta \gamma_{m}\right)_{\alpha} \mathfrak{e}_{P}^{m}+\frac{1}{3}\left(\theta \gamma^{m}\right)_{\alpha}\left(\theta \gamma_{m} \mathcal{X}_{P}\right)-\frac{1}{32}\left(\gamma^{p} \theta\right)_{\alpha}\left(\theta \gamma_{m n p} \theta\right) \mathfrak{f}_{P}^{m n}+\ldots\right) e^{k_{P} \cdot x}
$$

takes the same form as the linearized superfield $A_{\alpha}^{i}$ subject to $(2.11)[33,34]$,

$$
A_{\alpha}^{i}(x, \theta)=\left(\frac{1}{2}\left(\theta \gamma_{m}\right)_{\alpha} e_{i}^{m}+\frac{1}{3}\left(\theta \gamma^{m}\right)_{\alpha}\left(\theta \gamma_{m} \chi_{i}\right)-\frac{1}{32}\left(\gamma^{p} \theta\right)_{\alpha}\left(\theta \gamma_{m n p} \theta\right) f_{i}^{m n}+\ldots\right) e^{k_{i} \cdot x}
$$

The components $\mathfrak{e}_{P}^{m}, \mathcal{X}_{P}^{\alpha}, \mathfrak{f}_{P}^{m n}$ depend on the momenta $k_{i}^{m}$, polarizations $e_{i}^{m}$ and wavefunctions $\chi_{i}^{\alpha}$ of the gluons and gluinos encompassed in the multiparticle label $P=12 \ldots p$ and can be obtained from the recursions [15]

$$
\mathfrak{e}_{P}^{m}=\frac{1}{s_{P}} \sum_{X Y=P} \mathfrak{e}_{[X, Y]}^{m}, \quad \mathcal{X}_{P}^{\alpha}=\frac{1}{s_{P}} \sum_{X Y=P} \mathcal{X}_{[X, Y]}^{\alpha},
$$

where $\mathfrak{e}_{i}^{m} \equiv e_{i}^{m}$ and $\mathcal{X}_{i}^{\alpha} \equiv \chi_{i}^{\alpha}$ for a single-particle label as well as

$$
\begin{aligned}
\mathfrak{e}_{[X, Y]}^{m} & \equiv-\frac{1}{2}\left[\mathfrak{e}_{X}^{m}\left(k^{X} \cdot \mathfrak{e}^{Y}\right)+\mathfrak{e}_{n}^{X} \mathfrak{f}_{Y}^{m n}-\left(\mathcal{X}^{X} \gamma^{m} \mathcal{X}^{Y}\right)-(X \leftrightarrow Y)\right] \\
\mathcal{X}_{[X, Y]}^{\alpha} & \equiv \frac{1}{2}\left(k_{X}^{p}+k_{Y}^{p}\right) \gamma_{p}^{\alpha \beta}\left[\mathfrak{e}_{X}^{m}\left(\gamma_{m} \mathcal{X}_{Y}\right)_{\beta}-\mathfrak{e}_{Y}^{m}\left(\gamma_{m} \mathcal{X}_{X}\right)_{\beta}\right] .
\end{aligned}
$$

The non-linear component field-strength is given by

$$
\mathfrak{f}_{P}^{m n} \equiv k_{P}^{m} \mathfrak{e}_{P}^{n}-k_{P}^{n} \mathfrak{e}_{P}^{m}-\sum_{X Y=P}\left(\mathfrak{e}_{X}^{m} \mathfrak{e}_{Y}^{n}-\mathfrak{e}_{X}^{n} \mathfrak{e}_{Y}^{m}\right)
$$

and generalizes the single-particle instance $\mathfrak{f}_{i}^{m n} \equiv f_{i}^{m n}=k_{i}^{m} e_{i}^{n}-k_{i}^{n} e_{i}^{m}$ in (2.14).

The expressions in (2.16), (2.17) and (2.18) are obtained from the theta-independent terms of the superfields $\mathcal{A}_{P}^{m}, \mathcal{W}_{P}^{\alpha}, \mathcal{F}_{P}^{m n}$ evaluated at $x=0$ [15],

$$
\mathfrak{e}_{P}^{m} \equiv \mathcal{A}_{P}^{m}(0,0), \quad \mathcal{X}_{P}^{\alpha} \equiv \mathcal{W}_{P}^{\alpha}(0,0), \quad \mathfrak{f}_{P}^{m n} \equiv \mathcal{F}_{P}^{m n}(0,0)
$$

in the same way as $e_{i}^{m}, \chi_{i}^{\alpha}$ and $f_{i}^{m n}$ stem from the linearized superfields $A_{i}^{m}, W_{i}^{\alpha}, F_{i}^{m n}$. Accordingly, the recursions in (2.15) to (2.17) for $\mathfrak{e}_{P}^{m}$ and $\mathcal{X}_{P}^{\alpha}$ descend from the recursive construction of superspace Berends-Giele currents $\mathcal{A}_{P}^{m}, \mathcal{W}_{P}^{\alpha}, \mathcal{F}_{P}^{m n}$ described in [15].

Note that the transversality of the gluon and the Dirac equation of the gluino propagate as follows to the multiparticle level,

$$
\left(k_{P} \cdot \mathfrak{e}_{P}\right)=0, \quad k_{m}^{P}\left(\gamma^{m} \mathcal{X}_{P}\right)_{\alpha}=\sum_{X Y=P}\left[\mathfrak{e}_{X}^{m}\left(\gamma_{m} \mathcal{X}_{Y}\right)_{\alpha}-\mathfrak{e}_{Y}^{m}\left(\gamma_{m} \mathcal{X}_{X}\right)_{\alpha}\right]
$$

where transversality of $\mathfrak{e}_{P}^{m}$ is a peculiarity of the Lorentz gauge chosen in the derivation of the corresponding superspace Berends-Giele current $\mathcal{A}_{P}^{m}(x, \theta)[15]$. 


\subsection{The pure spinor superspace formula for SYM tree amplitudes}

Tree-level amplitudes in ten-dimensional SYM have been constructed in [6] from cohomology methods in pure spinor superspace [5]. Inspired by OPEs in string theory, the BRST-invariant superspace expression

$$
A^{\mathrm{SYM}}(1,2, \ldots, p, p+1)=\left\langle E_{12 \ldots p} M_{p+1}\right\rangle \equiv \sum_{X Y=12 \ldots p}\left\langle M_{X} M_{Y} M_{p+1}\right\rangle
$$

with the pole structure of a color-ordered $(p+1)$-point amplitude has been proposed and shown to reproduce known component expressions for various combinations of gluons and gluinos. BRST invariance of the superfields implies gauge-invariant and supersymmetric components. In $(2.21)$ the bracket $\langle\ldots\rangle$ instructs to pick up terms of order $\lambda^{3} \theta^{5}$ of the enclosed superfields [5], and the following shorthand has been used

$$
M_{P} \equiv \lambda^{\alpha} \mathcal{A}_{\alpha}^{P}(x, \theta)
$$

for contractions of the pure spinor $\lambda^{\alpha}$. At this point, we make use of the gauge choice in [15] where the theta-expansion (2.13) of the multiparticle superfield mimics the single-particle counterpart (2.14). In this way, the same $\lambda^{3} \theta^{5}$ correlators listed on appendix A of [35] govern both the three-point amplitude

$$
A^{\mathrm{SYM}}(1,2,3)=\left\langle M_{1} M_{2} M_{3}\right\rangle=\frac{1}{2} \mathfrak{e}_{1}^{m} \mathfrak{f}_{2}^{m n} \mathfrak{e}_{3}^{n}+\left(\mathcal{X}_{1} \gamma_{m} \mathcal{X}_{2}\right) \mathfrak{e}_{3}^{m}+\operatorname{cyclic}(123)
$$

and a generic multiparticle constituent of the $n$-point amplitudes (2.21),

$$
\left\langle M_{X} M_{Y} M_{Z}\right\rangle=\frac{1}{2} \mathfrak{e}_{X}^{m} \mathfrak{f}_{Y}^{m n} \mathfrak{e}_{Z}^{n}+\left(\mathcal{X}_{X} \gamma_{m} \mathcal{X}_{Y}\right) \mathfrak{e}_{Z}^{m}+\operatorname{cyclic}(X Y Z) \equiv \mathfrak{M}_{X, Y, Z}
$$

This makes the gluon and gluino components of an arbitrary $n$-point tree amplitude easily accessible through the recursion (2.15) to (2.18) for the components $\mathfrak{e}_{P}^{m}, \mathcal{X}_{P}^{\alpha}$ and $\mathfrak{f}_{P}^{m n}$. Using the component field-strength (2.18), it follows that the gluonic three-point amplitudes of the Berends-Giele and pure spinor formulæ match. In the following section, we will demonstrate that the same is true for an arbitrary number of external legs.

\section{The supersymmetric completion of the Berends-Giele formula}

In this section, the pure spinor superspace formula for ten-dimensional SYM tree amplitudes (2.21) will be shown to reduce ipsis litteris to the Berends-Giele formula (2.6) when restricted to its gluonic expansion. Given the supersymmetry of the pure spinor approach, we will use it to derive the supersymmetric completion of the Berends-Giele formula.

\subsection{Bosonic Berends-Giele current from superfields}

In a first step, the lowest components $\mathfrak{e}_{P}^{m}$ in the superfield (2.13) are demonstrated to reproduce the bosonic Berends-Giele currents in (2.1) once the fermions are decoupled, i.e.

$$
\left.\mathfrak{e}_{P}^{m}\right|_{\chi_{j}=0}=J_{P}^{m}
$$


Plugging the field-strength $\mathfrak{f}_{P}^{m n}(2.18)$ into the recursive definition of $\mathfrak{e}_{P}^{m}(2.15)$ leads to

$$
\begin{aligned}
2 s_{P} \mathfrak{e}_{P}^{m}= & -\sum_{X Y=P}\left[2 \mathfrak{e}_{X}^{m}\left(k_{X} \cdot \mathfrak{e}_{Y}\right)+k_{Y}^{m}\left(\mathfrak{e}_{X} \cdot \mathfrak{e}_{Y}\right)-\left(\mathcal{X}_{X} \gamma^{m} \mathcal{X}_{Y}\right)-(X \leftrightarrow Y)\right] \\
& +\sum_{X Y Z=P}\left[2\left(\mathfrak{e}_{X} \cdot \mathfrak{e}_{Z}\right) \mathfrak{e}_{Y}^{m}-\left(\mathfrak{e}_{X} \cdot \mathfrak{e}_{Y}\right) \mathfrak{e}_{Z}^{m}-\left(\mathfrak{e}_{Y} \cdot \mathfrak{e}_{Z}\right) \mathfrak{e}_{X}^{m}\right] .
\end{aligned}
$$

In absence of fermions, $\chi_{j}^{\alpha}=0$, the first line (3.2) yields the contribution of the cubic vertex (2.3) to the Berends-Giele current, and the second line due to the non-linear part of the field-strength $\mathfrak{f}_{P}^{m n}$ reproduces the quartic vertex (2.4). This is natural since the quartic interaction in the YM Lagrangian arises from the non-linear part of the field-strength. Together with the single-particle case $\mathfrak{e}_{i}^{m}=J_{i}^{m}=e_{i}^{m}$, the matching of (3.2) at $\chi_{j}^{\alpha}=0$ with the Berends-Giele recursion (2.1) completes the inductive proof of (3.1).

Also note that the recursion (2.17) for $\mathcal{X}_{P}^{\alpha}$ amounts to a resummation of Feynman diagrams incorporating both the fermion propagator $k_{m} \gamma_{\alpha \beta}^{m} / k^{2}$ and the cubic coupling of two fermions with a boson, in accordance with the Berends-Giele method [16] applied to ten-dimensional SYM theory.

\subsection{Supersymmetric Berends-Giele amplitude from the pure spinor formula}

The relation (3.1) between the ten-dimensional Berends-Giele current $\mathfrak{e}_{P}^{m}$ in superspace and its purely gluonic counterpart $J_{P}^{m}$ is now extended to their corresponding tree-level amplitudes: the pure spinor formula (2.21) versus the Berends-Giele formula (2.6).

To see the relation, note that (2.24) can be rewritten as

$$
\begin{aligned}
\left\langle M_{X} M_{Y} M_{Z}\right\rangle= & \left(\mathfrak{e}_{[X, Y]} \cdot \mathfrak{e}_{Z}\right)+\mathfrak{e}_{X}^{m}\left(\mathcal{X}_{Y} \gamma_{m} \mathcal{X}_{Z}\right)-\mathfrak{e}_{Y}^{m}\left(\mathcal{X}_{X} \gamma_{m} \mathcal{X}_{Z}\right) \\
& +\frac{1}{2} \sum_{R S=Z}\left[\left(\mathfrak{e}_{R} \cdot \mathfrak{e}_{X}\right)\left(\mathfrak{e}_{S} \cdot \mathfrak{e}_{Y}\right)-\left(\mathfrak{e}_{R} \cdot \mathfrak{e}_{Y}\right)\left(\mathfrak{e}_{S} \cdot \mathfrak{e}_{X}\right)\right],
\end{aligned}
$$

provided that transversality (2.20) and momentum conservation holds, $k_{X}^{m}+k_{Y}^{m}+k_{Z}^{m}=0$. In particular, when $Z \rightarrow p+1$ is a single-particle label associated with the $(p+1)^{\text {th }}$ massless leg, the deconcatenation terms in the second line of (3.3) vanish:

$$
\left\langle M_{X} M_{Y} M_{p+1}\right\rangle=\left(\mathfrak{e}_{[X, Y]} \cdot \mathfrak{e}_{p+1}\right)+\mathfrak{e}_{X}^{m}\left(\mathcal{X}_{Y} \gamma_{m} \mathcal{X}_{p+1}\right)-\mathfrak{e}_{Y}^{m}\left(\mathcal{X}_{X} \gamma_{m} \mathcal{X}_{p+1}\right) .
$$

Plugging the correlator (3.4) into the pure spinor superspace formula for tree-level SYM amplitudes (2.21) yields

$$
A^{\mathrm{SYM}}(1,2, \ldots p, p+1)=\sum_{X Y=12 \ldots p}\left[\left(\mathfrak{e}_{[X, Y]} \cdot \mathfrak{e}_{p+1}\right)+\mathfrak{e}_{X}^{m}\left(\mathcal{X}_{Y} \gamma_{m} \mathcal{X}_{p+1}\right)-\mathfrak{e}_{Y}^{m}\left(\mathcal{X}_{X} \gamma_{m} \mathcal{X}_{p+1}\right)\right]
$$

Alternatively, using (2.15) and (2.20) to identify $\mathfrak{e}_{12 \ldots p}^{m}$ and $\mathcal{X}_{12 \ldots p}^{\alpha}$, this can be written as

$$
A^{\mathrm{SYM}}(1,2, \ldots p, p+1)=s_{12 \ldots p}\left(\mathfrak{e}_{12 \ldots p} \cdot \mathfrak{e}_{p+1}\right)+k_{12 \ldots p}^{m}\left(\mathcal{X}_{12 \ldots p} \gamma_{m} \mathcal{X}_{p+1}\right) .
$$

In view of (3.1), the expression (3.6) reproduces the gluonic Berends-Giele formula [16] in absence of fermions,

$$
\left.A^{\mathrm{SYM}}(1,2 \ldots, p, p+1)\right|_{\chi_{j}=0}=s_{12 \ldots p}\left(J_{12 \ldots p} \cdot J_{p+1}\right)=A^{\mathrm{YM}}(1,2 \ldots, p, p+1),
$$


and additionally provides its supersymmetric completion. Note that the bosonic currents $\mathfrak{e}_{P}^{m}$ contain even powers of gluino wavefunctions $\chi_{i}^{\alpha}$ from the last term in (2.16) such as $s_{12} \mathfrak{e}_{12}^{m}=s_{12} J_{12}^{m}+\left(\chi_{1} \gamma^{m} \chi_{2}\right)$. Hence, both classes of terms on the right hand side of (3.6) contribute to fermionic amplitudes.

\subsection{Divergent propagators and their cancellation}

\subsubsection{In components}

From the definition (2.15) it follows that both of $\mathfrak{e}_{P}^{m}$ and $\mathcal{X}_{P}^{\alpha}$ in (3.6) are proportional to a divergent propagator since $s_{P}=0$ for a massless $(p+1)$-point amplitude. As well known from the Berends-Giele formula for gluons [16], this is compensated by the formally vanishing numerator containing $s_{P}=0$ in (2.6). The same is true for its supersymmetric completion derived in (3.6) since $k_{P}^{m}\left(\gamma_{m} \mathcal{X}_{p+1}\right)_{\alpha}=0$ using $k_{P}^{m}=-k_{p+1}^{m}$ and the massless Dirac equation. The interpretation is also the same; $s_{P}$ is the inverse of the bosonic propagator $1 / \partial^{2}$ while $k_{m}^{P} \gamma_{\alpha \beta}^{m}$ is the inverse of the fermion propagator $\partial_{m} \gamma_{\alpha \beta}^{m} / \partial^{2}$.

\subsubsection{In pure spinor superspace}

The supersymmetric way to cancel a divergent propagator relies on the action of the pure spinor BRST charge $Q \equiv \lambda^{\alpha} D_{\alpha}[5]$ on the currents $M_{P}[6]$,

$$
E_{P} \equiv Q M_{P}=\sum_{X Y=P} M_{X} M_{Y}
$$

The integration of schematic form $\left\langle\lambda^{3} \theta^{5}\right\rangle=1$ annihilates BRST-exact expressions [5]. Because the single-particle superfield $M_{p+1}$ is BRST closed, $Q M_{p+1}=0$, the superspace representation of tree-level amplitudes in (2.21) would be BRST exact $Q\left(M_{P} M_{p+1}\right)$ if the current $M_{P}$ was well defined in the phase space of $p+1$ massless particles [6]. However, $M_{P} \sim 1 / s_{P}$ and therefore the vanishing of $s_{P}$ prevents the amplitude from being BRST exact. Just like (3.5), the expression $\left\langle\sum_{X Y=P} M_{X} M_{Y} M_{p+1}\right\rangle$ does not contain any divergent propagator.

The assessment of BRST-exactness for a given superfield will play an important role in the derivation of BCJ relations in section 4.2.

\subsection{Short representations and BRST integration by parts}

At first sight the Berends-Giele formula (2.6) requires the $p$-current $J_{12 \ldots p}^{m}$ in the computation of the $(p+1)$-gluon amplitude. However, a diagrammatic method has been used by Berends and Giele in [17] to obtain "short" representations of bosonic amplitudes up to eight points which required no more than the four-current and led to manifestly cyclic formulæ for $A^{\mathrm{YM}}(1,2, \ldots, p+1)$. For example, the six-point amplitude was found to be

$$
\begin{aligned}
A^{\mathrm{YM}}(1,2, \ldots, 6)= & \frac{1}{2} s_{123} J_{123}^{m} J_{456}^{m}+\frac{1}{3}\left[J_{12}, J_{34}\right]^{m} J_{56}^{m} \\
& +\frac{1}{2}\left\{J_{1}, J_{23}, J_{4}\right\}^{m} J_{56}^{m}+\left\{J_{1}, J_{2}, J_{34}\right\}^{m} J_{56}^{m}+\operatorname{cyclic}(123456),
\end{aligned}
$$

and similar expressions were written for the seven- and eight-point amplitudes [17]. 
In the framework of pure spinor superspace, the multiplicity of currents can be shortened using integration by parts of the BRST charge. By (3.8), this amounts to

$$
\sum_{X Y=P}\left\langle M_{X} M_{Y} M_{Q}\right\rangle=\sum_{X Y=Q}\left\langle M_{P} M_{X} M_{Y}\right\rangle
$$

which has been used in [6] to cast the superspace formula (2.21) for $n$-point trees into a manifestly cyclic form without any current of multiplicity higher than $\frac{n}{2}$, e.g.

$$
A^{\mathrm{SYM}}(1,2, \ldots, 6)=\frac{1}{3}\left\langle M_{12} M_{34} M_{56}\right\rangle+\frac{1}{2}\left\langle M_{123}\left(M_{45} M_{6}+M_{4} M_{56}\right)\right\rangle+\operatorname{cyclic}(123456) .
$$

In terms of the components $\mathfrak{M}_{X, Y, Z}$ from the evaluation (2.24) of pure spinor superspace expressions, the component expressions for amplitudes of multiplicity $\leq 8$ are given by

$$
\begin{aligned}
A^{\mathrm{SYM}}(1,2, \ldots, 4)= & \frac{1}{2} \mathfrak{M}_{12,3,4}+\operatorname{cyclic}(12 \ldots 4) \\
A^{\mathrm{SYM}}(1,2, \ldots, 5)= & \mathfrak{M}_{12,3,45}+\operatorname{cyclic}(12 \ldots 5) \\
A^{\mathrm{SYM}}(1,2, \ldots, 6)= & \frac{1}{3} \mathfrak{M}_{12,34,56}+\frac{1}{2}\left(\mathfrak{M}_{123,45,6}+\mathfrak{M}_{123,4,56}\right)+\operatorname{cyclic}(12 \ldots 6) \\
A^{\mathrm{SYM}}(1,2, \ldots, 7)= & \mathfrak{M}_{123,45,67}+\mathfrak{M}_{1,234,567}+\operatorname{cyclic}(12 \ldots 7) \\
A^{\mathrm{SYM}}(1,2, \ldots, 8)= & \frac{1}{2}\left(\mathfrak{M}_{1234,567,8}+\mathfrak{M}_{1234,56,78}+\mathfrak{M}_{1234,5,678}\right) \\
& +\mathfrak{M}_{123,456,78}+\operatorname{cyclic}(12 \ldots 8),
\end{aligned}
$$

see [6] for the nine- and ten-point analogues. Given the recursive nature of the definitions of $\mathfrak{e}_{P}^{m}, \mathfrak{f}_{P}^{m n}$ and $\mathcal{X}_{P}^{\alpha}$, the full component expansion of the above amplitudes is readily available and reproduce the results available on the website [36].

Note that the manipulations leading to (3.4) rely on a single-particle current $M_{p+1}$ and therefore do not apply to the $\mathfrak{M}_{X, Y, Z}$ in (3.12).

\subsection{The generating series of tree-level amplitudes}

The way how component amplitudes (3.6) of SYM descend from the pure spinor superspace expression (2.21) can be phrased in the language of generating series. The solution

$$
\mathbb{V} \equiv \lambda^{\alpha} \mathbb{A}_{\alpha}=\sum_{i} M_{i} t^{i}+\sum_{i, j} M_{i j} t^{i} t^{j}+\sum_{i, j, k} M_{i j k} t^{i} t^{j} t^{k}+\cdots
$$

of the non-linear SYM equations (2.9) generates color-dressed SYM amplitudes via ${ }^{5}[18]$

$$
\frac{1}{3} \operatorname{Tr}\langle\mathbb{V} \mathbb{V}\rangle=\sum_{n=3}^{\infty} \frac{n-2}{n} \sum_{i_{1}, i_{2}, \ldots, i_{n}} \operatorname{Tr}\left(t^{i_{1}} t^{i_{2}} \ldots t^{i_{n}}\right) A^{\mathrm{SYM}}\left(i_{1}, i_{2}, \ldots, i_{n}\right)
$$

\footnotetext{
${ }^{5}$ The representations of SYM amplitudes generated by $\operatorname{Tr}\langle\mathbb{V} \mathbb{V}\rangle$ are related to (2.21) by BRST integration by parts (3.10).
} 
Note from (2.19) that $\mathfrak{e}_{P}^{m}, \mathcal{X}_{P}^{\alpha}$ and $\mathfrak{f}_{P}^{m n}$ are just the $\theta=0$ components of the corresponding generating series $\mathbb{A}^{m}, \mathbb{W}^{\alpha}$ and $\mathbb{F}^{m n}$. Therefore (2.24) implies that

$$
\begin{aligned}
\frac{1}{3} \operatorname{Tr}\langle\mathbb{V} \mathbb{V}\rangle & =\frac{1}{4} \operatorname{Tr}\left(\left[\mathbb{A}_{m}, \mathbb{A}_{n}\right] \mathbb{F}^{m n}\right)+\left.\operatorname{Tr}\left(\mathbb{W} \gamma^{m} \mathbb{A}_{m} \mathbb{W}\right)\right|_{\theta=0} \\
& =\left.\operatorname{Tr}\left(\frac{1}{4} \mathbb{F}_{m n} \mathbb{F}^{m n}+\left(\mathbb{W} \gamma^{m} \nabla_{m} \mathbb{W}\right)\right)\right|_{\theta=0}
\end{aligned}
$$

In passing to the second line of (3.15), we have used the massless Dirac equation $\nabla_{m} \gamma_{\alpha \beta}^{m} \mathbb{W}^{\beta}=0$ as well as the field equation $\partial_{m} \mathbb{F}^{m n}=\left[\mathbb{A}_{m}, \mathbb{F}^{m n}\right]+\gamma_{\alpha \beta}^{n}\left\{\mathbb{W}^{\alpha}, \mathbb{W}^{\beta}\right\}$ and discarded a total derivative to rewrite $\left(\partial_{m} \mathbb{A}_{n}\right) \mathbb{F}^{m n}=-\mathbb{A}_{n}\left(\left[\mathbb{A}_{m}, \mathbb{F}^{m n}\right]+\gamma_{\alpha \beta}^{n}\left\{\mathbb{W}^{\alpha}, \mathbb{W}^{\beta}\right\}\right)$. The factor $1 / 3$ on the left-hand side of (3.15) offsets the sum over three terms that results from the cyclic symmetry of the trace.

It is interesting to observe that the generating series of tree-level amplitudes (3.15) matches the ten-dimensional SYM Lagrangian evaluated on the generating series of (nonlocal) Berends-Giele currents in superspace: $\mathbb{F}^{m n}(x, 0)$ and $\mathbb{W}^{\alpha}(x, 0)$.

\section{BCJ relations from the cohomology of pure spinor superspace}

In this section, we prove that the BCJ relations [19] among partial SYM amplitudes follow from the vanishing of certain BRST-exact expressions in pure spinor superspace and find a closed formula for them. A closely related property of tree amplitudes is the possibility to express the complete kinematic dependence in terms of $(n-2)$ ! master numerators through a sequence of Jacobi-like relations [19]. A superspace representation of such master numerators was given in [21], and we will provide a compact component evaluation along the lines of the previous section.

\subsection{Kleiss-Kuijf relations from symmetries of Berends-Giele currents}

For completeness, we start by revisiting from a superspace perspective the Kleiss-Kuijf (KK) relations among color-ordered amplitudes [37], firstly proven in [38].

The KK relations are conveniently described in the Berends-Giele framework. To see this, recall that the superspace currents $\mathcal{K}_{P} \in\left\{\mathcal{A}_{\alpha}^{P}, \mathcal{A}_{P}^{m}, \mathcal{W}_{P}^{\alpha}, \mathcal{F}_{P}^{m n}\right\}$ satisfy the symmetry property [9]

$$
\mathcal{K}_{A ш B}=0, \quad \forall A, B \neq \emptyset,
$$

see appendix B of [15] for a proof. The symmetry (4.1) of course also holds for thetaindependent components $\left\{\mathfrak{e}_{P}^{m}, \mathcal{X}_{P}^{\alpha}, \mathfrak{f}_{P}^{m n}\right\}$ of $\mathcal{K}_{P}$, see (2.19). Since the currents $\mathfrak{e}_{P}^{m}$ reduce to $J_{P}^{m}$ via (3.1), this is consistent with the symmetry $J_{A \uplus B}^{m}=0, \forall A, B \neq \emptyset$ derived by Berends and Giele in [27]. The symmetry (4.1) together with the identity ${ }^{6}$

$$
\mathcal{K}_{B 1 A}-(-1)^{|B|} \mathcal{K}_{1\left(A ш B^{T}\right)}=-\sum_{X Y=B}(-1)^{|X|} \mathcal{K}_{X^{T} \uplus(Y 1 A)}-(-1)^{|B|} \mathcal{K}_{B^{T} \uplus(1 A)},
$$

\footnotetext{
${ }^{6}$ Incidentally, the identity (4.2) shows the equivalence between the statements given in equation (2) of [39] and Theorem 2.2 of [40].
} 


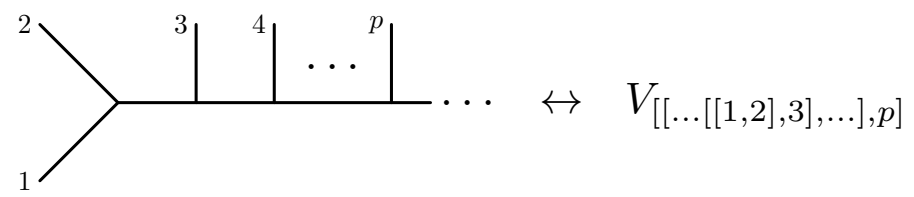

Figure 1. The tree diagram with an off-shell leg is represented by the local superfield (4.6).

where $B^{T}$ denotes the reversal of the word $B$, lead to an alternative form of (4.1),

$$
\mathcal{K}_{B 1 A}-(-1)^{|B|} \mathcal{K}_{1\left(A ш B^{T}\right)}=0 .
$$

Since $E_{P} \equiv Q M_{P}$ generalizes (4.3) to $\mathcal{K}_{P} \rightarrow E_{P}$, the tree-level amplitude representa$\operatorname{tion}^{7}$ (3.6) $A_{12 \ldots n}=\left\langle E_{12 \ldots n-1} M_{n}\right\rangle$ immediately yields the Kleiss-Kuijf relations

$$
A_{C 1 B n}-(-1)^{|C|} A_{1\left(B ш C^{T}\right) n}=\left\langle\left(E_{C 1 B}-(-1)^{|C|} E_{1\left(B ш C^{T}\right)}\right) M_{n}\right\rangle=0,
$$

which reduce the number of independent color-ordered amplitudes to $(n-2)$ ! [37].

\subsection{BCJ relations from the BRST cohomology}

\subsubsection{Berends-Giele currents in BCJ gauge}

There is a method to construct Berends-Giele currents from quotients of local superfields $K_{[P, Q]}$ by Mandelstam invariants whose precise form follows from an intuitive mapping with cubic graphs (or planar binary trees) $[9,15]$. For example, the Berends-Giele currents associated with the local superfield $V_{[P, Q]} \equiv \lambda^{\alpha} A_{\alpha}^{[P, Q]}$ up to multiplicity four are given by

$$
\begin{aligned}
& M_{12}^{\mathrm{BCJ}}=\frac{V_{[1,2]}}{s_{12}}, \quad M_{123}^{\mathrm{BCJ}}=\frac{V_{[[1,2], 3]}}{s_{12} s_{123}}+\frac{V_{[1,[2,3]]}}{s_{23} s_{123}}, \\
& M_{1234}^{\mathrm{BCJ}}=\frac{1}{s_{1234}}\left(\frac{V_{[[1,2], 3], 4]}}{s_{12} s_{123}}+\frac{V_{[[1,[2,3]], 4]}}{s_{23} s_{123}}+\frac{V_{[[1,2],[3,4]]}}{s_{12} s_{34}}+\frac{V_{[1,[2,[3,4]]]}}{s_{34} s_{234}}+\frac{V_{[1,[[2,3], 4]]}}{s_{23} s_{234}}\right) .
\end{aligned}
$$

As discussed in a companion paper [15], one can perform a multiparticle gauge transformation (denoted BCJ gauge) which enforces the superfields

$$
V_{123 \ldots p} \equiv V_{[[\ldots[[1,2], 3], \ldots], p]}
$$

in (4.5) with diagrammatic interpretation shown in figure 1 to satisfy the Lie symmetries of nested commutators $\left[\left[\ldots\left[\left[t^{1}, t^{2}\right], t^{3}\right], \ldots\right], t^{p}\right]$, e.g.

$$
V_{12}+V_{21}=0, \quad V_{123}+V_{231}+V_{312}=0 .
$$

Moreover, BCJ gauge allows to reduce any other topology of bracketings to the master topology (4.6) by a sequence of Jacobi-like identities

$$
V_{\ldots[[P, Q], R] \ldots}+V_{\ldots[[Q, R], P] \ldots}+V_{\ldots[[R, P], Q] \ldots}=0, \quad \text { e.g. } V_{[[1,2],[3,4]]}=V_{1234}-V_{1243} .
$$

\footnotetext{
${ }^{7}$ We omit the superscript from $A^{\mathrm{SYM}}$ and write the labels as a subscript to avoid cluttering.
} 
Hence, the Berends-Giele current $M_{12 \ldots p}^{\mathrm{BCJ}}$ can be expanded in terms of the $(p-1)$ ! independent permutations of $V_{12 \ldots}$. This is the same number of independent components as left by the Berends-Giele symmetry (4.1) (here for $\mathcal{K}_{12 \ldots p} \rightarrow M_{12 \ldots p}^{\mathrm{BCJ}}$ ). As a crucial feature of Berends-Giele currents in BCJ gauge, there is an invertible mapping between the local superfields $V_{12 \ldots p}$ and $M_{12 \ldots p}^{\mathrm{BCJ}}$. More explicitly, for multiplicity $p \leq 4$ one can use (4.6) and (4.8) to invert (4.5) and obtain

$$
\begin{aligned}
& V_{12}= s_{12} \\
& M_{12}^{\mathrm{BCJ}}, \quad V_{123}=s_{12}\left(s_{23} M_{123}^{\mathrm{BCJ}}-s_{13} M_{213}^{\mathrm{BCJ}}\right) \\
& V_{1234}=s_{12}[ s_{23} s_{34} M_{1234}^{\mathrm{BCJ}}-s_{13} s_{34} M_{2134}^{\mathrm{BCJ}}+s_{14} s_{23} M_{3214}^{\mathrm{BCJ}}-s_{13} s_{24} M_{3124}^{\mathrm{BCJ}} \\
&\left.+s_{23} s_{24}\left(M_{1234}^{\mathrm{BCJ}}+M_{1243}^{\mathrm{BCJ}}\right)-s_{13} s_{14}\left(M_{2134}^{\mathrm{BCJ}}+M_{2143}^{\mathrm{BCJ}}\right)\right] .
\end{aligned}
$$

The generalization to arbitrary rank can be read off from the formula [13]

$$
\frac{V_{12 \ldots p}}{z_{12} z_{23} \cdots z_{p-1, p}}+\operatorname{perm}(2, \ldots, p)=\prod_{k=2}^{p} \sum_{m=1}^{k-1} \frac{s_{m k}}{z_{m k}} M_{12 \ldots p}^{\mathrm{BCJ}}+\operatorname{perm}(2, \ldots, p),
$$

using partial fraction relations ${ }^{8}$ among the denominators made of $z_{i j} \equiv z_{i}-z_{j}$.

It is important to stress that the left-hand sides in (4.9) are local expressions; all the kinematic poles in Mandelstam invariants cancel out from the linear combinations of currents on the right-hand side. The poles cancel only when the superfields are in the BCJ gauge. As we will see below, this fact can be exploited to derive the BCJ relations [19] among color-ordered amplitudes.

\subsubsection{Four- and five-point BCJ relations}

We shall now connect superfields in BCJ gauge with BCJ relations among partial SYM amplitudes. At the four- and five-point level, one multiplies the local expressions in (4.9) by a single-particle $V_{n}$ (which is BRST closed) and uses the vanishing of BRST-exact expressions under the pure spinor bracket prescription $\langle\ldots\rangle[5]$. For example,

$$
\frac{V_{123}}{s_{12}}=s_{23} M_{123}^{\mathrm{BCJ}}-s_{13} M_{213}^{\mathrm{BCJ}} \Rightarrow 0=\left\langle Q\left(\frac{V_{123}}{s_{12}} V_{4}\right)\right\rangle=\left\langle\left(s_{23} E_{123}^{\mathrm{BCJ}}-s_{13} E_{213}^{\mathrm{BCJ}}\right) V_{4}\right\rangle
$$

with $E_{P}^{\mathrm{BCJ}} \equiv Q M_{P}^{\mathrm{BCJ}}$ corresponds to the four-point ${ }^{9}$ BCJ relation [19] by (2.21),

$$
0=s_{23} A^{\mathrm{SYM}}(1,2,3,4)-s_{13} A^{\mathrm{SYM}}(2,1,3,4) .
$$

Note that the BCJ gauge for the local superfields is a crucial requirement in this derivation - in a generic gauge, $s_{23} M_{123}-s_{13} M_{213}$ would be an ill-defined expression containing divergent propagators of the form $1 / s_{123}$ and the BRST triviality of $\left(s_{23} E_{123}-s_{13} E_{213}\right) V_{4}$ would no longer be guaranteed.

\footnotetext{
${ }^{8}$ Note that $Z_{12 \ldots p-1, p} \equiv 1 /\left(z_{12} z_{23} \ldots z_{p-1, p}\right)$ satisfies $Z_{A ш B}=0, \forall A, B \neq \emptyset$.

${ }^{9}$ The three-point BCJ relation $0=s_{12} A^{\mathrm{SYM}}(1,2,3)$ following from $s_{12}=0$ can be formally derived via $0=\left\langle Q V_{12} V_{3}\right\rangle=s_{12}\left\langle V_{1} V_{2} V_{3}\right\rangle$.
} 
Similarly, the identities

$$
\begin{aligned}
\frac{V_{1234}}{s_{12} s_{123}}+\frac{V_{3214}}{s_{23} s_{123}} & =s_{34} M_{1234}^{\mathrm{BCJ}}+s_{14} M_{3214}^{\mathrm{BCJ}}-s_{24}\left(M_{1324}^{\mathrm{BCJ}}+M_{3124}^{\mathrm{BCJ}}\right) \\
\frac{V_{1234}-V_{1243}}{s_{12} s_{34}} & =s_{23} M_{1234}^{\mathrm{BCJ}}-s_{13} M_{2134}^{\mathrm{BCJ}}-s_{24} M_{1243}^{\mathrm{BCJ}}+s_{14} M_{2143}^{\mathrm{BCJ}}
\end{aligned}
$$

derived from (4.9) with manifestly well-defined left-hand side imply the BCJ relations [19]

$$
\begin{aligned}
0=\left\langle Q\left(\frac{V_{1234}}{s_{12} s_{123}}+\frac{V_{3214}}{s_{23} s_{123}}\right) V_{5}\right\rangle= & s_{34} A^{\mathrm{SYM}}(1,2,3,4,5)+s_{14} A^{\mathrm{SYM}}(3,2,1,4,5) \\
& -s_{24}\left[A^{\mathrm{SYM}}(1,3,2,4,5)+A^{\mathrm{SYM}}(3,1,2,4,5)\right] \\
0=\left\langle Q\left(\frac{V_{1234}-V_{1243}}{s_{12} s_{34}}\right) V_{5}\right\rangle= & s_{23} A^{\mathrm{SYM}}(1,2,3,4,5)-s_{13} A^{\mathrm{SYM}}(2,1,3,4,5) \\
& -s_{24} A^{\mathrm{SYM}}(1,2,4,3,5)+s_{14} A^{\mathrm{SYM}}(2,1,4,3,5) .
\end{aligned}
$$

Even though the above derivation relies on the choice of BCJ gauge, the subamplitudes in the resulting BCJ relations are independent on the multiparticle gauge for the currents $M_{P}$. This can be seen from the non-linear gauge invariance in the generating series (3.14) of the amplitude formula (2.21).

\subsubsection{Higher-point BCJ relations}

Along the same lines, one can verify in a basis of $V_{P}$ that the expression [9]

$$
M_{S[A, B]}^{\mathrm{BCJ}} \equiv \sum_{i=1}^{|A|} \sum_{j=1}^{|B|}(-1)^{i-j+|A|-1} s_{a_{i} b_{j}} M_{\left(a_{1} a_{2} \ldots a_{i-1} ш a_{|A|} a_{|A|-1} \ldots a_{i+1}\right) a_{i} b_{j}\left(b_{j-1} \ldots b_{2} b_{1} ш b_{j+1} \ldots b_{|B|}\right)}^{\mathrm{BCJ}}
$$

with $A=a_{1} a_{2} \ldots a_{|A|}$ and $B=b_{1} b_{2} \ldots b_{|B|}$ does not have any pole in $s_{A B}$. One can therefore identify the following BRST-exact combinations of $(|A|+|B|+1)$-point amplitudes,

$$
\begin{aligned}
0 & =(-1)^{|A|-1}\left\langle Q\left(M_{S[A, B]}^{\mathrm{BCJ}} M_{n}\right)\right\rangle \\
& =\sum_{i=1}^{|A|} \sum_{j=1}^{|B|}(-1)^{i-j} s_{a_{i} b_{j}}\left\langle E_{\left(a_{1} a_{2} \ldots a_{i-1} ш a_{|A|} a_{|A|-1} \ldots a_{i+1}\right) a_{i} b_{j}\left(b_{j-1} \ldots b_{2} b_{1} ш b_{j+1} \ldots b_{|B|}\right)}^{\mathrm{BCJ}} M_{n}\right\rangle \\
& =\sum_{i=1}^{|A|} \sum_{j=1}^{|B|}(-1)^{i-j} s_{a_{i} b_{j}} A^{\mathrm{SYM}}\left(\left(a_{1} \ldots a_{i-1} ш a_{|A|} \ldots a_{i+1}\right), a_{i}, b_{j},\left(b_{j-1} \ldots b_{1} ш b_{j+1} \ldots b_{|B|}\right), n\right),
\end{aligned}
$$

which all boil down to BCJ relations in some representation [19, 23, 24, 26]. For the singleparticle choice $A=1$ along with $B=2,3,4, \ldots,(n-1),(4.16)$ reduces to the fundamental BCJ relations

$$
\begin{aligned}
0= & -\left\langle Q\left(M_{S[1,234 \ldots(n-1)]}^{\mathrm{BCJ}} M_{n}\right)\right\rangle \\
= & s_{12} A^{\mathrm{SYM}}(2,1,3, \ldots, n)+\left(s_{12}+s_{13}\right) A^{\mathrm{SYM}}(2,3,1,4, \ldots, n) \\
& +\cdots+\left(s_{12}+s_{13}+\ldots+s_{1, n-1}\right) A^{\mathrm{SYM}}(2,3, \ldots, n-1,1, n),
\end{aligned}
$$

which are well-known to leave $(n-3)$ ! independent subamplitudes [19, 23, 24, 26]. 


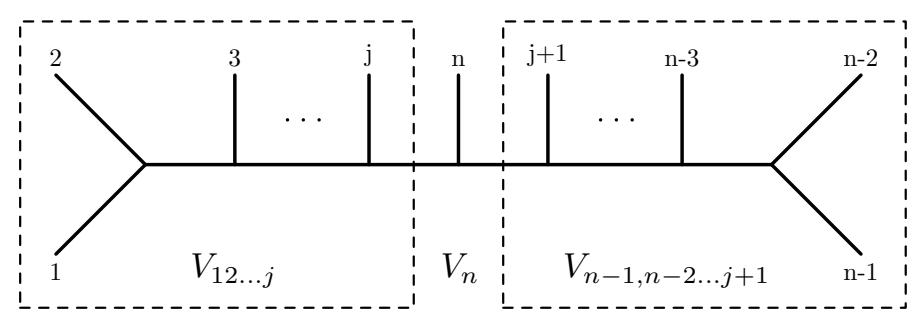

Figure 2. The $(n-2)$ ! half-ladder diagrams with legs 1 and $n-1$ attached to opposite endpoints encode the complete kinematic dependence in a BCJ representation.

\subsection{Component form of BCJ numerators}

The initial derivation of BCJ relations in [19] relied on the duality between color and kinematics, i.e. the existence of particular representations of tree amplitudes. The functions of polarizations and momenta associated with the cubic graphs in such a "BCJ representation" are assumed to obey the same Jacobi identities as the color factors made of structure constants $f^{a b c}$ of the gauge group. As a consequence, the complete information on polarizations and momenta reside in $(n-2)$ ! master graphs which can be chosen to be the half-ladder diagrams with fixed endpoints 1 and $n-1$ as depicted in figure 2 and arbitrary permutations of the remaining legs $2,3, \ldots, n-2$ and $n$.

An explicit realization of the BCJ duality for tree-level amplitudes was given in [21] based on the tree amplitudes of the pure spinor superstring. The master graphs in the figure were associated with local kinematic numerators ${ }^{10}\left\langle V_{12 \ldots j} V_{n-1, n-2 \ldots j+1} V_{n}\right\rangle$ labeled by $j=1,2, \ldots, n-2$ along with the $(n-3)$ ! permutations of the legs $2,3, \ldots, n-2$. The kinematic factors for any other graph can be reached by a sequence of Jacobi relations, and this representation agrees with the field-theory limit of the open superstring amplitude, i.e. yields the right SYM amplitude.

The techniques of [15] (in particular the discussion of BCJ/HS gauge) give rise to a compact formula for their components,

$$
\left\langle V_{A} V_{B} V_{C}\right\rangle=\frac{1}{2} e_{A}^{m} f_{B}^{m n} e_{C}^{n}+\left(\chi_{A} \gamma_{m} \chi_{B}\right) e_{C}^{m}+\operatorname{cyclic}(A B C),
$$

whose form is completely analogous to (2.24). The constituents $e_{A}^{m}, f_{A}^{m n}$ and $\chi_{A}^{\alpha}$ of (4.18) are local multiparticle polarizations and will be explained below.

\subsubsection{Local multiparticle polarizations}

The discussion of recursion relations for local superfields given in [15] has a direct counterpart for their multiparticle polarizations $e_{A}^{m}, f_{A}^{m n}$ and $\chi_{A}^{\alpha}$ which constitute their thetaindependent terms. The setup starts with a recursive definition for local multiparticle polarizations $\hat{e}_{A}^{m}, \hat{f}_{A}^{m n}$ and $\hat{\chi}_{A}^{\alpha}$ whose labels do not satisfy the symmetries of a Lie algebra, for example $\hat{e}_{123}^{m}+\hat{e}_{231}^{m}+\hat{e}_{312}^{m} \neq 0$ (their hatted notation is a reminder of this symmetry failure). However, non-linear gauge variations of their multiparticle superfields can be exploited to find a gauge where the symmetries are indeed satisfied.

\footnotetext{
${ }^{10}$ Note that the precursors of $V_{12 \ldots p}$ were denoted by $T_{12 \ldots p}$ in [21].
} 
The recursive definition of the hatted components is given by

$$
\begin{aligned}
& \hat{e}_{12 \ldots p}^{m}=-\frac{1}{2}\left[\hat{e}_{12 \ldots p-1}^{m}\left(k_{12 \ldots p-1} \cdot \hat{e}_{p}\right)+\hat{e}_{12 \ldots p-1}^{n} \hat{f}_{p}^{m n}-\left(\hat{\chi}_{12 \ldots p-1} \gamma^{m} \hat{\chi}_{p}\right)-(12 \ldots p-1 \leftrightarrow p)\right] \\
& \hat{\chi}_{12 \ldots p}^{\alpha}=\frac{1}{2} k_{12 \ldots p}^{n} \gamma_{n}^{\alpha \beta}\left[\hat{e}_{12 \ldots p-1}^{m}\left(\gamma_{m} \hat{\chi}_{p}\right)_{\beta}-(12 \ldots p-1 \leftrightarrow p)\right],
\end{aligned}
$$

and it starts with $\hat{e}_{i}^{m}=e_{i}^{m}$ and $\hat{\chi}_{i}^{\alpha}=\chi_{i}^{\alpha}$. The local field-strength is defined by

$$
\hat{f}_{m n}^{12 \ldots p} \equiv k_{m}^{12 \ldots p} \hat{e}_{n}^{12 \ldots p}-k_{n}^{12 \ldots p} \hat{e}_{m}^{12 \ldots p}+\sum_{j=2}^{p} \sum_{\delta \in P\left(\beta_{j}\right)}\left(k_{12 \ldots j-1} \cdot k_{j}\right) \hat{e}_{[n}^{12 \ldots j-1,\{\delta\}} \hat{e}_{m]}^{j,\left\{\beta_{j} \backslash \delta\right\}},
$$

with shorthand $\beta_{j}=\{j+1, j+2, \ldots, p\}$ and $P\left(\beta_{j}\right)$ denoting the power set of $\beta_{j}$, e.g.

$$
\begin{aligned}
& \hat{f}_{1}^{m n}=f_{1}^{m n}=k_{1}^{m} e_{1}^{n}-k_{1}^{n} e_{1}^{m}, \quad \hat{f}_{12}^{m n}=f_{12}^{m n}=k_{12}^{m} e_{12}^{n}-k_{12}^{n} e_{12}^{m}-s_{12} e_{1}^{[m} e_{2}^{n]} \\
& \hat{f}_{123}^{m n}=k_{123}^{m} \hat{e}_{123}^{n}-k_{123}^{n} \hat{e}_{123}^{m}-\left(s_{13}+s_{23}\right) e_{12}^{[n} e_{3}^{m]}-s_{12}\left(e_{1}^{[n} e_{23}^{m]}-e_{2}^{[n} e_{13}^{m]}\right) .
\end{aligned}
$$

Up to and including multiplicity $p=2$, the multiparticle polarizations in the BCJ numerators (4.18) agree with their hatted counterparts in (4.19),

$$
\begin{aligned}
& e_{12}^{m}=\hat{e}_{12}^{m}=e_{2}^{m}\left(e_{1} \cdot k_{2}\right)-e_{1}^{m}\left(e_{2} \cdot k_{1}\right)+\frac{1}{2}\left(k_{1}^{m}-k_{2}^{m}\right)\left(e_{1} \cdot e_{2}\right)+\left(\chi_{1} \gamma^{m} \chi_{2}\right) \\
& \chi_{12}^{\alpha}=\hat{\chi}_{12}^{\alpha}=\frac{1}{2} k_{12}^{p} \gamma_{p}^{\alpha \beta}\left[e_{1}^{m}\left(\gamma_{m} \chi_{2}\right)_{\beta}-e_{2}^{m}\left(\gamma_{m} \chi_{1}\right)_{\beta}\right],
\end{aligned}
$$

while multiplicities $p \geq 3$ require redefinitions $\hat{h}_{12 \ldots p}$ starting with

$$
e_{123}^{m}=\hat{e}_{123}^{m}-k_{123}^{m} \hat{h}_{123}, \quad \chi_{123}^{\alpha}=\hat{\chi}_{123}^{\alpha} .
$$

The redefinition of $\hat{e}_{123}^{m}$ in (4.23) ensures the Lie symmetry $e_{123}^{m}+e_{231}^{m}+e_{312}^{m}=0$. At multiplicity $p=4$, we have

$$
\begin{aligned}
& e_{1234}^{m}=\hat{e}_{1234}^{m}+\left(k_{123} \cdot k_{4}\right) \hat{h}_{123} e_{4}^{m}-\left(k_{12} \cdot k_{3}\right) \hat{h}_{124} e_{3}^{m}-\left(k_{1} \cdot k_{2}\right)\left(\hat{h}_{134} e_{2}^{m}-\hat{h}_{234} e_{1}^{m}\right)-k_{1234}^{m} \hat{h}_{1234} \\
& \chi_{1234}^{\alpha}=\hat{\chi}_{1234}^{\alpha}+\left(k_{123} \cdot k_{4}\right) \hat{h}_{123} \chi_{4}^{\alpha}-\left(k_{12} \cdot k_{3}\right) \hat{h}_{124} \chi_{3}^{\alpha}-\left(k_{1} \cdot k_{2}\right)\left(\hat{h}_{134} \chi_{2}^{\alpha}-\hat{h}_{234} \chi_{1}^{\alpha}\right),
\end{aligned}
$$

and the rank-five example can be extracted from [15] as will be explained shortly. The scalar correction terms $\hat{h}_{12 \ldots p}$ in (4.23) and (4.24) can be reduced to building blocks

$$
h_{A, B, C} \equiv \frac{1}{4} e_{A}^{m} f_{B}^{m n} e_{C}^{n}+\frac{1}{2}\left(\chi_{A} \gamma_{m} \chi_{B}\right) e_{C}^{m}+\operatorname{cyclic}(A B C)
$$

made of multiparticle polarizations of lower multiplicity $\leq p-2$ via

$$
\begin{aligned}
3 \hat{h}_{123} \equiv & h_{1,2,3} \\
4 \hat{h}_{1234} \equiv & h_{12,3,4}+h_{34,1,2}-\frac{1}{2} h_{1,2,3}\left(k_{123} \cdot e_{4}\right) \\
& +\frac{1}{6}\left[h_{1,3,4}\left(k_{134} \cdot e_{2}\right)-h_{2,3,4}\left(k_{234} \cdot e_{1}\right)-h_{1,2,4}\left(k_{124} \cdot e_{3}\right)\right] .
\end{aligned}
$$

Once the redefinition $e_{12 \ldots p}^{m}=\hat{e}_{12 \ldots p}^{m}+\ldots$ for the multiparticle polarization has been performed, the corresponding "unhatted" field-strength relevant for the BCJ numerators in (4.18) is obtained completely analogously to (4.20),

$$
f_{m n}^{12 \ldots p} \equiv k_{m}^{12 \ldots p} e_{n}^{12 \ldots p}-k_{n}^{12 \ldots p} e_{m}^{12 \ldots p}+\sum_{j=2}^{p} \sum_{\delta \in P\left(\beta_{j}\right)}\left(k_{12 \ldots j-1} \cdot k_{j}\right) e_{[n}^{12 \ldots j-1,\{\delta\}} e_{m]}^{j,\left\{\beta_{j} \backslash \delta\right\}}
$$




\subsubsection{Higher multiplicity}

As already mentioned, the above redefinitions of $\hat{e}_{12 \ldots p}^{m}, \hat{\chi}_{12 \ldots p}^{\alpha}$ and $\hat{f}_{12 \ldots p}^{m n}$ descend from the superspace discussion in section 3 of [15]. In particular, the corrections $h_{A, B, C}$ defined in (4.25) are the $\theta=0$ component of a local superfield $H_{A, B, C}(x, \theta)$ which was completely specified up to multiplicity five in [15]. So the full expressions of $e_{12345}^{m}, \chi_{12345}^{\alpha}$ and $f_{12345}^{m n}$ are readily available.

At the same time, there is no obstruction to pushing these recursive constructions even further, leading to local multiparticle polarizations $e_{P}^{m}, \chi_{P}^{\alpha}$ and $f_{P}^{m n}$ of higher multiplicity. Therefore, together with the central formula (4.18) for local components, the discussion in this section provides access to the supersymmetric components of the local BCJ-satisfying numerators of [21] in a recursive fashion.

\section{Conclusion and outlook}

In this work, we have extracted and streamlined component information from tree-level scattering amplitudes in pure spinor superspace. The results are based on simplified thetaexpansions for multiparticle superfields of ten-dimensional SYM which are attained via non-linear gauge transformations in a companion paper [15]. More specifically:

- The $n$-point tree-level amplitude derived in [6] from locality, supersymmetry and gauge invariance is shown to reproduce the Berends-Giele formula, and the supersymmetrization by fermionic component amplitudes is worked out.

- BCJ relations are derived from the decoupling of BRST-exact expressions in pure spinor superspace.

- Kinematic tree-level numerators [21] satisfying the BCJ duality between color and kinematics are translated into components.

The resulting ten-dimensional component amplitudes together with their BCJ representations and dimensional reductions will have a broad range of applications. With appropriate truncations of the gluon and gluino components, they are suitable to determine $D$-dimensional unitarity cuts in a variety of theories including QCD, see e.g. [41-43] and references therein.

It would be interesting to relate the multiparticle polarizations in the component form of the BCJ numerators to the approach of [44]. In that reference, formally vanishing nonlocal terms are added to the Yang-Mills Lagrangian to automatically produce BCJ numerators. The interplay between Lagrangians and generating series of kinematic factors might shed further light on the superfield redefinitions in [15] underlying our BCJ numerators.

\section{Acknowledgments}

We would like to express our gratitude to Seungjin Lee for collaboration on the closely related paper [15] and for numerous insightful discussions. We are also indebted to JohnJoseph Carrasco for valuable comments on a draft of this article. CRM wishes to acknowledge support from NSF grant number PHY 1314311 and the Paul Dirac Fund. We 
acknowledge support by the European Research Council Advanced Grant No. 247252 of Michael Green. OS is grateful to DAMTP in Cambridge for kind hospitality during various stages of this project, and OS additionally thanks the IAS in Princeton for kind hospitality during completion of this work. CRM is grateful to AEI in Potsdam for the warm hospitality during intermediate stages of this work and for partial financial support.

Open Access. This article is distributed under the terms of the Creative Commons Attribution License (CC-BY 4.0), which permits any use, distribution and reproduction in any medium, provided the original author(s) and source are credited.

\section{References}

[1] E. Witten, Twistor-Like Transform in Ten-Dimensions, Nucl. Phys. B 266 (1986) 245 [INSPIRE].

[2] L. Brink, J.H. Schwarz and J. Scherk, Supersymmetric Yang-Mills Theories, Nucl. Phys. B 121 (1977) 77 [INSPIRE].

[3] P.S. Howe, Pure spinors lines in superspace and ten-dimensional supersymmetric theories, Phys. Lett. B 258 (1991) 141 [Addendum ibid. B 259 (1991) 511] [INSPIRE].

[4] P.S. Howe, Pure spinors, function superspaces and supergravity theories in ten-dimensions and eleven-dimensions, Phys. Lett. B 273 (1991) 90 [INSPIRE].

[5] N. Berkovits, Super Poincaré covariant quantization of the superstring, JHEP 04 (2000) 018 [hep-th/0001035] [INSPIRE].

[6] C.R. Mafra, O. Schlotterer, S. Stieberger and D. Tsimpis, A recursive method for SYM n-point tree amplitudes, Phys. Rev. D 83 (2011) 126012 [arXiv: 1012.3981] [INSPIRE].

[7] C.R. Mafra and O. Schlotterer, Towards one-loop SYM amplitudes from the pure spinor BRST cohomology, Fortsch. Phys. 63 (2015) 105 [arXiv:1410.0668] [INSPIRE].

[8] C.R. Mafra and O. Schlotterer, Two-loop five-point amplitudes of super Yang-Mills and supergravity in pure spinor superspace, JHEP 10 (2015) 124 [arXiv: 1505. 02746] [INSPIRE].

[9] C.R. Mafra and O. Schlotterer, Multiparticle SYM equations of motion and pure spinor BRST blocks, JHEP 07 (2014) 153 [arXiv: 1404.4986] [INSPIRE].

[10] C.R. Mafra, Pure Spinor Superspace Identities for Massless Four-point Kinematic Factors, JHEP 04 (2008) 093 [arXiv: 0801.0580] [INSPIRE].

[11] C.R. Mafra, Simplifying the Tree-level Superstring Massless Five-point Amplitude, JHEP 01 (2010) 007 [arXiv:0909.5206] [INSPIRE].

[12] C.R. Mafra, O. Schlotterer, S. Stieberger and D. Tsimpis, Six Open String Disk Amplitude in Pure Spinor Superspace, Nucl. Phys. B 846 (2011) 359 [arXiv:1011.0994] [InSPIRE].

[13] C.R. Mafra, O. Schlotterer and S. Stieberger, Complete N-Point Superstring Disk Amplitude I. Pure Spinor Computation, Nucl. Phys. B 873 (2013) 419 [arXiv:1106.2645] [InSPIRE].

[14] C.R. Mafra and O. Schlotterer, The Structure of n-Point One-Loop Open Superstring Amplitudes, JHEP 08 (2014) 099 [arXiv: 1203.6215] [INSPIRE].

[15] S. Lee, C.R. Mafra and O. Schlotterer, Non-linear gauge transformations in D $=10 S Y M$ theory and the BCJ duality, arXiv:1510.08843 [INSPIRE]. 
[16] F.A. Berends and W.T. Giele, Recursive Calculations for Processes with n Gluons, Nucl. Phys. B 306 (1988) 759 [INSPIRE].

[17] F.A. Berends, W.T. Giele and H. Kuijf, Exact and Approximate Expressions for Multi-Gluon Scattering, Nucl. Phys. B 333 (1990) 120 [InSPIRE].

[18] C.R. Mafra and O. Schlotterer, Solution to the nonlinear field equations of ten dimensional supersymmetric Yang-Mills theory, Phys. Rev. D 92 (2015) 066001 [arXiv:1501.05562] [INSPIRE].

[19] Z. Bern, J.J.M. Carrasco and H. Johansson, New Relations for Gauge-Theory Amplitudes, Phys. Rev. D 78 (2008) 085011 [arXiv:0805.3993] [INSPIRE].

[20] J.J.M. Carrasco, Gauge and Gravity Amplitude Relations, arXiv:1506.00974 [INSPIRE].

[21] C.R. Mafra, O. Schlotterer and S. Stieberger, Explicit BCJ Numerators from Pure Spinors, JHEP 07 (2011) 092 [arXiv: 1104.5224] [INSPIRE].

[22] C.R. Mafra, PSS: A FORM Program to Evaluate Pure Spinor Superspace Expressions, arXiv:1007. 4999 [INSPIRE].

[23] N.E.J. Bjerrum-Bohr, P.H. Damgaard and P. Vanhove, Minimal Basis for Gauge Theory Amplitudes, Phys. Rev. Lett. 103 (2009) 161602 [arXiv:0907.1425] [INSPIRE].

[24] S. Stieberger, Open 83 Closed vs. Pure Open String Disk Amplitudes, arXiv:0907.2211 [INSPIRE].

[25] C.R. Mafra, O. Schlotterer and S. Stieberger, Complete N-Point Superstring Disk Amplitude II. Amplitude and Hypergeometric Function Structure, Nucl. Phys. B 873 (2013) 461 [arXiv:1106.2646] [INSPIRE].

[26] Y.-X. Chen, Y.-J. Du and B. Feng, A Proof of the Explicit Minimal-basis Expansion of Tree Amplitudes in Gauge Field Theory, JHEP 02 (2011) 112 [arXiv:1101.0009] [INSPIRE].

[27] F.A. Berends and W.T. Giele, Multiple Soft Gluon Radiation in Parton Processes, Nucl. Phys. B 313 (1989) 595 [inSPIRE].

[28] A.A. Rosly and K.G. Selivanov, On amplitudes in selfdual sector of Yang-Mills theory, Phys. Lett. B 399 (1997) 135 [hep-th/9611101] [INSPIRE].

[29] A.A. Rosly and K.G. Selivanov, Gravitational SD perturbiner, hep-th/9710196 [INSPIRE].

[30] K.G. Selivanov, Postclassicism in tree amplitudes, hep-th/9905128 [INSPIRE].

[31] K.G. Selivanov, On tree form-factors in (supersymmetric) Yang-Mills theory, Commun. Math. Phys. 208 (2000) 671 [hep-th/9809046] [INSPIRE].

[32] W.A. Bardeen, Selfdual Yang-Mills theory, integrability and multiparton amplitudes, Prog. Theor. Phys. Suppl. 123 (1996) 1 [INSPIRE].

[33] J.P. Harnad and S. Shnider, Constraints and field equations for ten-dimensional super Yang-Mills theory, Commun. Math. Phys. 106 (1986) 183 [INSPIRE].

[34] G. Policastro and D. Tsimpis, $R^{4}$, purified, Class. Quant. Grav. 23 (2006) 4753 [hep-th/0603165] [INSPIRE].

[35] N. Berkovits and C.R. Mafra, Some Superstring Amplitude Computations with the Non-Minimal Pure Spinor Formalism, JHEP 11 (2006) 079 [hep-th/0607187] [INSPIRE].

[36] C.R. Mafra and O. Schlotterer, PSS: From pure spinor superspace to components, http://www.damtp.cam.ac.uk/user/crm66/SYM/pss.html. 
[37] R. Kleiss and H. Kuijf, Multi-Gluon Cross-sections and Five Jet Production at Hadron Colliders, Nucl. Phys. B 312 (1989) 616 [InSPIRE].

[38] V. Del Duca, L.J. Dixon and F. Maltoni, New color decompositions for gauge amplitudes at tree and loop level, Nucl. Phys. B 571 (2000) 51 [hep-ph/9910563] [inSPIRE].

[39] M. Schocker, Lie elements and Knuth relations, Canad. J. Math. 56 (2004) 871 [math/0209327].

[40] R. Ree, Lie elements and an algebra associated with shuffles, Ann. Math. 68 (1958) 210.

[41] R.K. Ellis, W.T. Giele, Z. Kunszt and K. Melnikov, Masses, fermions and generalized D-dimensional unitarity, Nucl. Phys. B 822 (2009) 270 [arXiv:0806.3467] [InSPIRE].

[42] S. Davies, One-Loop QCD and Higgs to Partons Processes Using Six-Dimensional Helicity and Generalized Unitarity, Phys. Rev. D 84 (2011) 094016 [arXiv: 1108.0398] [INSPIRE].

[43] S. Badger, H. Frellesvig and Y. Zhang, A Two-Loop Five-Gluon Helicity Amplitude in QCD, JHEP 12 (2013) 045 [arXiv:1310.1051] [INSPIRE].

[44] M. Tolotti and S. Weinzierl, Construction of an effective Yang-Mills Lagrangian with manifest BCJ duality, JHEP 07 (2013) 111 [arXiv: 1306.2975] [INSPIRE]. 